\title{
ROLF HUISGEN'S CONTRIBUTION TO ORGANIC CHEMISTRY, EMPHASIZING 1,3-DIPOLAR CYCLOADDITIONS
}

\author{
Reiner Sustmann \\ Institut für Organische Chemie der Universität Essen, D-45117 \\ Essen, Germany
}

Heterocycles devotes a special issue to Rolf Huisgen in 1995 , the year where he celebrates his $75^{\text {th }}$ birthday. Heterocyclic Chemistry has indeed been the most important "adventure playground" of his research activities although his contributions to Organic Chemistry encompass a number of quite different areas. His monumental investigations on 1,3-dipolar cycloadditions have led to a new way of synthesizing many heterocyclic compounds. It is characteristic for Rolf Huisgen that these synthetic applications resulted from more fundamental investigations on the development and the elucidation of the mechanisms of new reactions. Thus, synthesis evolved in a natural way from of a physical organic approach to chemistry. It is a splendid idea to acknowledge the achievements of Rolf Huisgen in modern heterocyclic chemistry by this issue of Heterocycles and it is a pleasure for me to provide a short summary of his contributions to organic chemistry, emphasizing in particular 1,3-dipolar cycloadditions.

Rolf Huisgen studied at a time where, at least in Germany, emphasis in organic chemistry was given to isolation, structure elucidation, and synthesis of naturally occurring compounds. He began his scientific career with a topic from natural products chemistry under the guidance of Heinrich Wieland, one of the giants in organic chemistry in the first half of this century. The thesis, completed in 1943, describes studies on strychnos alkaloids, in particular degradation studies on vomicine. 
The conditions for a scientific career were unfavorable during and shortly after world war II. Nonetheless, Rolf Huisgen completed his PhD-studies in record time and habilitated, the habilitation thesis being the prerequisite for an academic career, already in 1947 at the age of 27. It dealt with angular versus linear fusion of heterorings, starting from $\beta$-substituted naphthalenes and quinolines. Shortly thereafter, he was offered a position as professor extraordinarius at the University of Tübingen. In 1952, at the age of 32 , he succeeded Heinrich Wieland, his academic teacher, as professor of organic chemistry at the University of Munich. Rolf Huisgen describes this event modestly in his recent book "The Adventure playground of Mechanisms and Novel Reactions" in the series on Profiles, Pathways, and Dreams 1): "I well understood that I received the chair at Munich, rich in tradition, not as a reward for scientific merits but rather as a trust for the future." Time has shown that the Bavarian minister of education took the proper decision.

Ever since 1952 Rolf Huisgen has been present at the forefront of organic chemistry. In Germany he introduced physical organic chemistry, an area which originated mainly in the anglo-american countries. This approach to chemistry seems to have fitted Rolf Huisgen's thinking perfectly. He used the methods of physical organic chemistry to develop his own way of choosing, analyzing, and tackling organic-chemical problems. Possessing a high intellectual capacity, combined with the capability to comprehend a chemical situation almost instantaneously and, furthermore, being a master in oral and written formulation, he attracted many young and talented chemists. In "Rolf Huisgen und sein Arbeitskreis (R.H. and his research group)"2), prepared at the occasion of his retirement from teaching in 1988, Siegfried Hünig writes ${ }^{3}$ ): "He had a kind of pacemaker function for organic chemistry in Germany after 1945". In the same volume Paul Schleyer notes 4 ): "Huisgen established physical organic chemistry in Germany after 1945 not only through the model character of his distinctive and exceptionally fine research, but also through the many coworkers who, both in academia and industry, continue to exemplify his approach to chemistry.

During the course of more than 50 years Rolf Huisgen made important contributions to a great number of different areas in organic chemistry. Up to now he has reported the results of his investigations in more than 500 publications and continues his activities. It is impossible to discuss all 
of his research subjects within the scope of this contribution. I have to leave out almost completely some of them, not meaning that they are less significant. Rather, I shall concentrate on 1,3-dipolar cycloadditions. This is the area which has had the highest impact on heterocyclic chemistry and which Rolf Huisgen himself considers his most important contribution to organic chemistry. I quote from his scientific selfportrait of 1994 " The adventure playground of mechanisms and novel reactions"1). "With 94 full papers, 109 communications, and 28 review articles on various aspects, 1,3-dipolar cycloadditions constitute my most extensive research effort; approximately 80 full papers are still to be written".

\section{1,3-Dipolar Cycloadditions}

Diazo compounds attracted Rolf Huisgen's interest at an early stage of his scientific work. He got intrigued by $\mathrm{N}$-nitrosoacylamides and their products of thermal decomposition in aromatic solvents and started own experimental studies on aromatic and aliphatic $\mathrm{N}$-nitrosoacylamıdes. The investigation of their rearrangement to diazoesters, their dissociation in either aromatic or aliphatic diazonium salts, and the subsequent formation of products via radical or ionic pathways are landmarks in Rolf Huisgen's early work. They reveal his typical approach of using kinetic and product studies to derive a detailed picture of a reaction. He was convinced that only the combination of both tools leads to reliable conclusions. The devotion to kinetics and the conviction of its power to solve mechanistic problems gave rise to an article on the application of kinetics in "Methoden der Organischen Chemie (Houben-Weyl) in 1954. This article was a "must" for every student joining his group. I remenber that students even before they had finished the diploma examination used to have a look at this article which was written for the practitioner

One example may suffice to demonstrate the elegance of the investigations in $\mathrm{N}$-nitrosoacylamide chemistry (scheme 1). The reaction of $\mathrm{N}$-nitrosomethylbenzamide was shown to lead to methyl benzoate, presumably via methyldiazonium benzoate as an intermediate. The question arose to which extent diazomethane might be involved in this reaction? Does the methyldiazonium ion deprotonate under these conditions? A competition experiment was set up in which $\mathrm{N}$ - 
nitrosomethylbenzamide reacted in the presence of 4-nitrobenzoic acid and N-nitrosomethyl-4nitrobenzamide in the presence of benzoic acid. It could be shown that both $\mathrm{N}$-nitrosoacylamides led to a mixture of methyl benzoate and methyl 4-nitrobenzoate in a ratio of $1: 4$. The conclusion that in both experiments the two carboxylic acids react with one and the same intermediate, derived from different precursors, was confirmed by the observation that diazomethane, the proposed intermediate, prepared by an independent route reacted with a mixture of the same two acids to a mixture of methy benzoate and methyl 4-nitrobenzoate in the same ratio as in the decomposition of the $\mathrm{N}$-nitrosoacylamides. Rolf Huisgen devised further experiments to identify the nature of the intermediate. In $\mathrm{H} / \mathrm{D}$ exchange studies on $\mathrm{N}$-nitrosomethylbenzamide with an excess of $\mathrm{C}_{6} \mathrm{H}_{5}-$ $\mathrm{CO}_{2} \mathrm{D}$ it was shown that incorporation of more than one equivalent of deuterium in methylbenzoate is most convincingly explained by invoking diazomethane as an intermediate.

Scheme 1

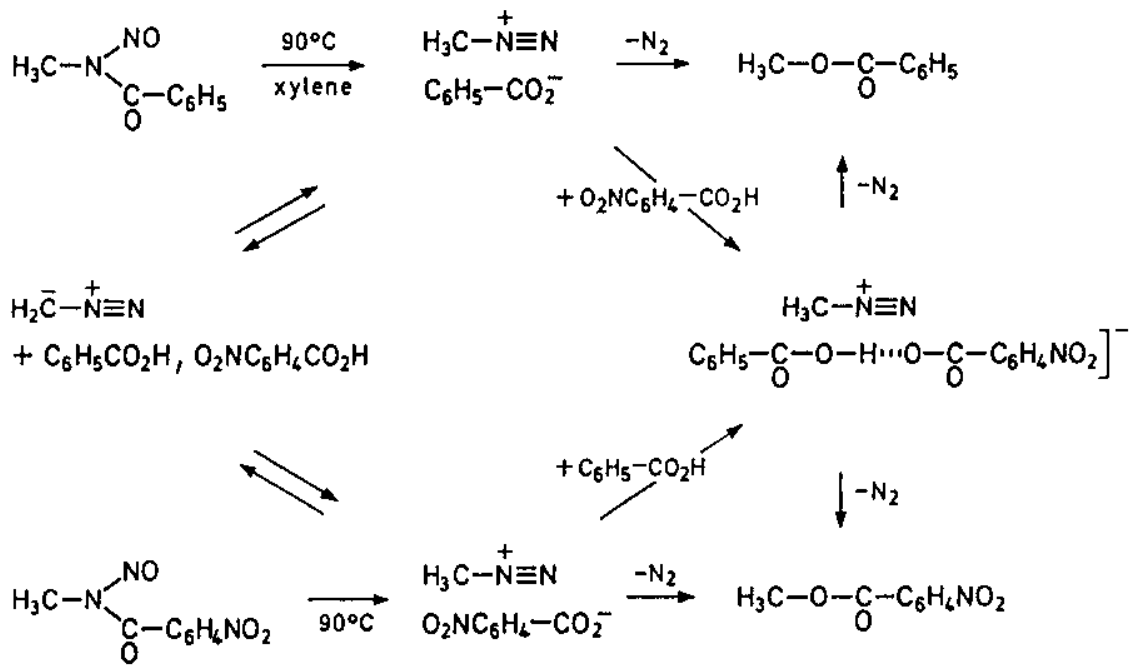

In a 1955 review Rolf Huisgen summarized the chemistry of diazoalkanes. His intimate familiarity with investigations which had been performed among others by so famous chemists as Theodor 
Curtius, Hans von Pechmann, and Eduard Buchner had influenced his own work in this area and seems to have been instrumental for the development of the concept of 1,3-dipolar cycloadditions.

The reactions of diazoalkanes with aromatic diazonium ions and with electrophilic azo compounds occupy a prominent place among these investigations. It was, for instance, established that 4nitrobenzenediazonium chloride combines with ethyl diazoacetate or diazoacetophenone to give $\alpha$ chlorohydrazones (scheme 2). These compounds were later used as convenient precursors for the nitrile imine skeleton through hydrogen chloride elimination by tertiary amines.

Scheme 2

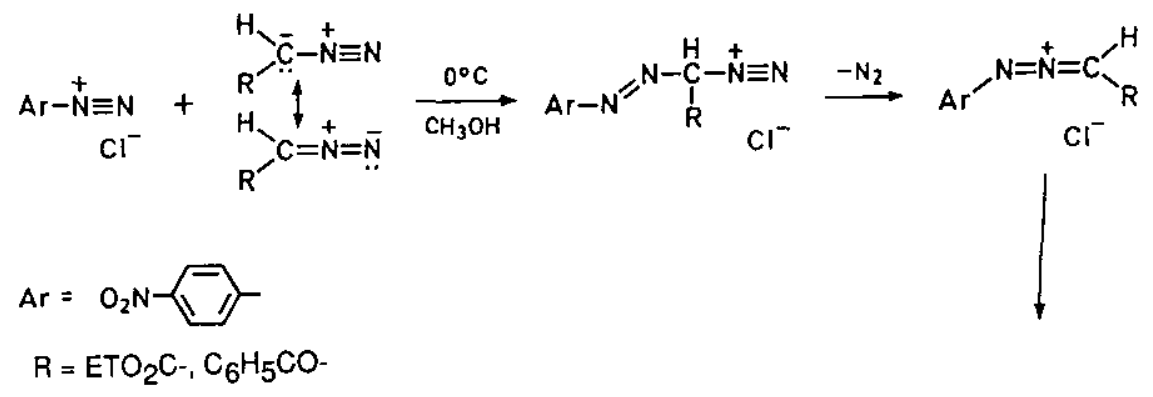<smiles>[Z19]N/N=C(/Cl)[R]([H])=[Al]N=NC([R])Cl</smiles>

Reactions of electrophilic azo compounds with diazoalkanes must have paved the ground for the discovery of the concept of 1,3-dipolar cycloadditions. 9-diazofluorene and 4-chlorobenzene-antidiazocyanide gave $93 \%$ of a compound for which the $x$-ray analysis revealed later the presence of the structural unit of an azomethine imine (scheme 3). At the moment of isolation it was not identified as a 1,3-dipole which might be capable of cycloaddition to unsaturated $\pi$-systems. 
Scheme 3

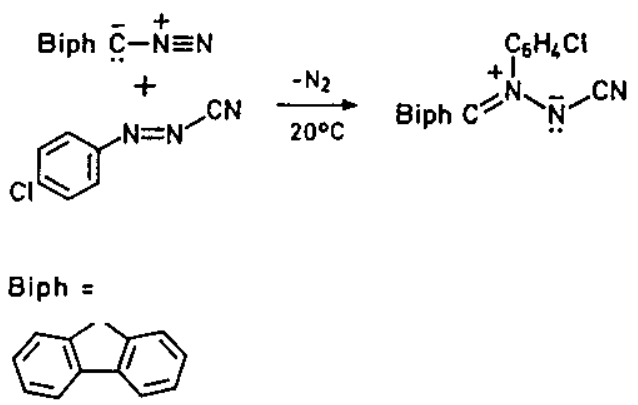

The dehydrohalogenation route to 1,3-dipoles from suitable precursors proved to be a facile way to prepare the corresponding 3,4-dihydroisoquinolinium $\mathrm{N}$-arylimides (A) shown in scheme 4 . The red azomethine imine dimerizes reversibly or forms an adduct in the presence of methanol $(B)$. Both, the dimer and the methanol adduct B, are precursors for the 1,3-dipole and yield cycloadducts (C) in the presence of olefinic or acetylenic substrates. Interestingly, the cycloaddition turned out to proceed regardless of the electronic nature of the unsaturated $\pi$-system, whether it is an enamine, a vinyl ether, a simple alkene, an $\alpha, \beta$-unsaturated carbonyl compound, or a nitrile.

Scheme 4

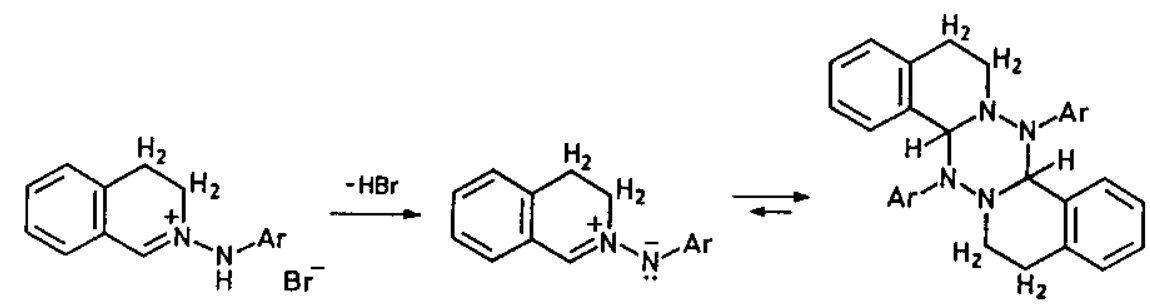

A<smiles>CCNN1CCc2ccccc2C1(O)OC</smiles>

B

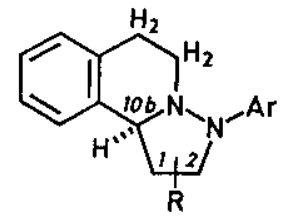

C 
The chemistry of azoles constituted another adventure playground of Rolf Huisgen in the time preceding 1,3-dipolar chemistry. The elegant work on the identification of pentazoles in the reaction of aromatic diazonium salts with azides fell into this period. The discovery of 1,3-diphenylnitrile imine, generated by thermolysis or photolysis of 2,5-diphenyltetrazole (scheme 5) was made at this time. Its capability to to undergo cycloaddition, leading to pyrazolines and pyrazoles (A in scheme 5 ) initiated a boom in 1,3-dipolar chemistry.

Scheme 5
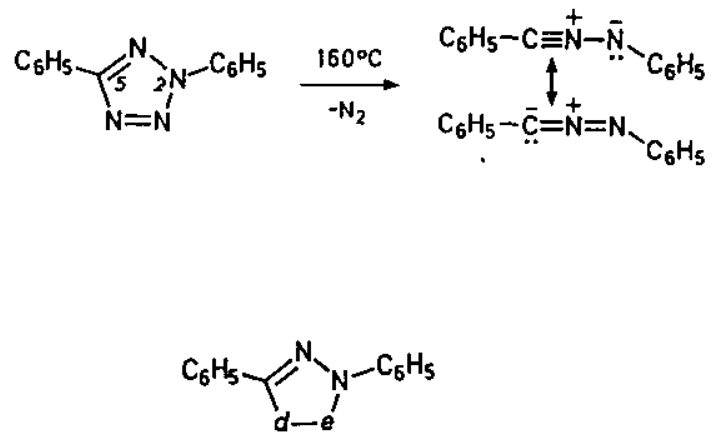

A

In 1960 Rolf Huisgen published the concept of 1,3-dipolar cycloadditions. During the investigations on azomethine imines and nitrile imines common bonding features became apparent when qualitative valence bond theory was used. Resonance structures could be drawn which unveiled immediately the similarity between azomethine imines and nitrile imines. Indeed, a preconception had already appeared in the 1955 review on diazoalkane chemistry where the five resonance structures, typical for 1,3-dipoles, had been introduced to describe the diazoalkane skeleton. Concurrently it was realized that aromatic azides for which reactions with double bonds were reported to give five-membered heterocyclic rings contained the bonding system of 1,3-dipoles.

In his unique, systematic, and perfectionist manner Rolf Huisgen developed and presented the classification of 1,3-dipoles with $\mathrm{C}, \mathrm{N}$, and $\mathrm{O}$ as center atoms (scheme 6). Allowing systems with or without an additional double bond, 1,3-dipoles of the propargyl-allenyl - and of the allyl type were 
obtained (scheme 7), altogether 18 different systems. Nine of these 18 were unknown previously and cycloadditions had been observed with only five of them.

Scheme 6

\begin{tabular}{|c|c|c|c|}
\hline \multicolumn{2}{|c|}{ Nitrilium Betaines } & \multicolumn{2}{|c|}{ Diazonium Betaines } \\
\hline$-\mathrm{C} \equiv \stackrel{+}{\mathrm{N}}-\overline{\mathrm{C}}<$ & Nitrile ylides & $\mathrm{N} \equiv \stackrel{+}{\mathrm{N}}-\overrightarrow{\mathrm{C}}<$ & Dlazoalkanes \\
\hline$-C \equiv \stackrel{+}{N^{-}}-\bar{N}^{-}$ & Nitrile imines & $N \equiv \stackrel{+}{N}-\overline{N^{-}}-$ & Azides \\
\hline$-\mathrm{C} \equiv \stackrel{+}{\mathrm{N}}-\overline{0}$ & Nitrile oxides & $\mathrm{N} \equiv \stackrel{+}{\mathrm{N}}-\overline{0}$ & Dinitrogen oxide \\
\hline \multicolumn{4}{|c|}{ 1,3-Dipoles of Allyl Type } \\
\hline \multicolumn{2}{|c|}{ Nitrogen Function as Middle Center } & Oxygen Atom & as Middle Center \\
\hline$>C=N_{1}^{+}-\bar{C}<$ & Azomethine ylides & $>c=\stackrel{+}{0}-\bar{c}<<$ & Carbonyl ylides \\
\hline$>C=N_{1}^{+}-\vec{N}^{-}$ & Azomethine imines & $>c=\stackrel{+}{0}-\vec{N}^{\prime}$ & Carbonyl imines \\
\hline$>C=N_{1}^{+}-0$. & Nitrones & $>c=\stackrel{+}{0}-\overline{0}$ & Carbonyl oxides \\
\hline$-N=N_{1}^{+}-N^{-}$ & Azimines & $-N=\stackrel{+}{0}-\bar{N}-$ & Nitrosimines \\
\hline$-N=N_{i}^{+}-\bar{O}$ & Azoxy Compounds & $-N=\stackrel{+}{0}-\overline{0}$ & Nitrosoxides \\
\hline $0=N_{1}^{+}-\overrightarrow{0}$ & Nitro Compounds & $0=\stackrel{+}{0}-\overline{0}$ & Ozone \\
\hline
\end{tabular}

Scheme 7

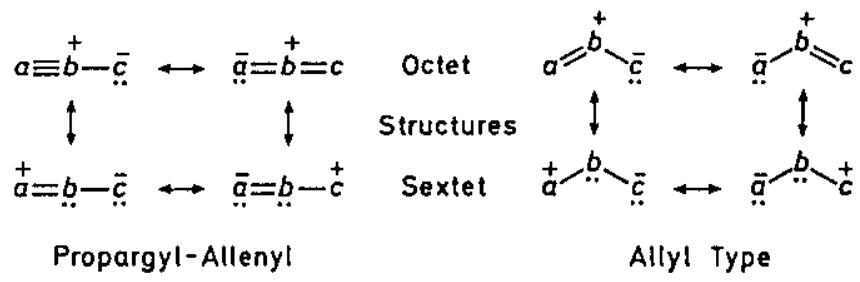

In the Centenary lecture in London in 1960 Rolf Huisgen described the excitement which had seized his laboratory: "In our work on 1,3-dipolar cycloadditions, in the first year one coworker was engaged 
in preliminary exploration. At the beginning of the second year two more people joined the effort. Now, in the third year, seventeen coworkers reap the harvest and contribute to further expansion of this reaction".

Obviously, the realization of the possible existence of as much as thirteen systems which might undergo 1,3-dipolar cycloadditions prompted a systematic search. Azomethine imines and nitrle imines had been instrumental for the development of the concept. Soon the first azomethine ylide (A in scheme 8) was generated by deprotonation of 3,4-dihydro-N-4-nitrobenzylisoquinolinium bromide with triethylamine; nitrones ( $\mathbf{B}$ in scheme 8 ) were another class of 1,3-dipoles to be studied systematically.

\section{Scheme 8}<smiles>[X][N+]1=Cc2ccccc2CC1</smiles><smiles>[R][N+]([O-])=Cc1ccccc1</smiles>

$$
\mathrm{X}=\mathrm{CH}-\mathrm{C}_{6} \mathrm{H}_{4} \mathrm{NO}_{2}-(4)
$$

In the early days it was more important to show the validity of the general concept than to pay attention to the substitution pattern of the 1,3-dipoles. Practical aspects about their generation were important. Many of the early 1,3-dipoles had aromatic substituents. Synthetic aspects and the anticipation that such groups might stabilize 1,3-dipoles through conjugation must have been the reason. Thırty years later, the wealth of synthetic applications, as displayed, for instance, in the two volumes of "1,3-dipolar cycloaddition chemistry", edited by A. Padwa, has clearly demonstrated that many different substitution patterns are possible, thus making available an incredible number of differently substituted five-membered heterocycles. 
It would have been surprising if mechanistic considerations had not played an important role in the development of the field from the beginning. Rate measurements on cycloadditions of diazoalkanes, carried out in Munich in 1958, contributed to the formulation of the concept. Soon, systematic kinetic investigations were started for cycloadditions of phenyl azide, C-phenyl-N-methylnitrone, benzonitrile oxide, C,C-biphenylene- $\mathrm{N}$-(4-chlorophenyl)- $\mathrm{N}$-cyanoazomethine imine and sydnones. These broad studies revealed interesting similarities and differences in the reactive behavior of 1,3-dipoles. The results implicated a common underlying mechanistic scheme. Together with the stereospecificity of the cycloaddition and the low dependence of the rates on solvent polarity they supported a concerted mechanism as the best description.

In his most cited review on 1,3-dipolar cycloadditions of 1963 in Angewandte Chemie Rolf Huisgen developed a qualitative molecular orbital description for the mechanism which should later be confirmed by rigorous quantum-mechanical calculations on model reactions. The 1,3-dipole and the dipolarophile were placed in two parallel planes in order to enable a $\sigma$-type overlap of the $\pi$-systems (scheme 9). At the same time it was pointed out that a 4-electron allylanion-type $\pi$-system was the reactive part of the 1,3-dipole.

\section{Scheme 9}

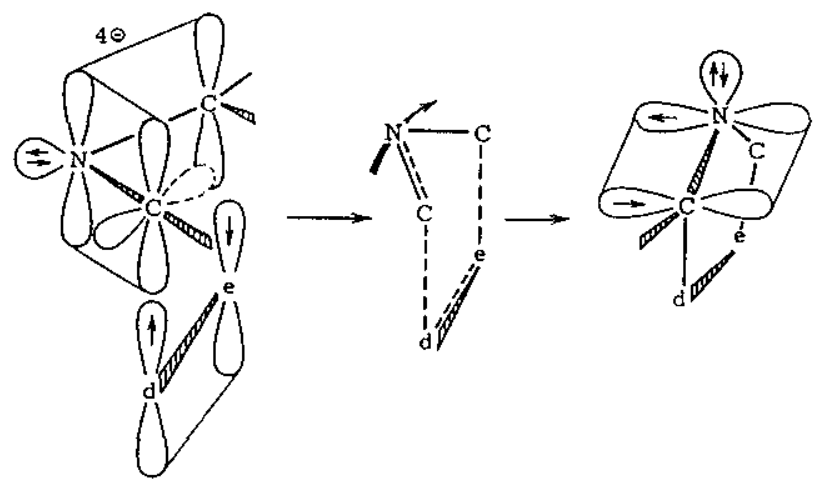

Huisgen's mechanistic representation gained strong theoretical support when in 1965 Woodward and Hoffmann proposed the rules of conservation of orbital symmetry for the Diels-Alder reaction and other polyene cycloadditions. Immediately Rolf Huisgen grasped the importance of these results. 
An analogous orbital and state correlation diagram was derived for 1,3-dipolar cycloadditions in the Huisgen group. The complete correspondence of the Diels-Alder cycloaddition and 1,3-dipolar cycloadditions was established. According to the Woodward-Hoffmann nomenclature both types of cycloaddition are allowed concerted processes when they occur in $(\pi 4 s+\pi 2 s)$ fashion.

Model calculations, so high their level may be, and also orbital symmetry considerations, so elegant and appealing they are, cannot claim to describe the behavior of all reactive systems in 1,3-dipolar or other cycloadditions properly. The substitution pattern of both reactive partners can influence and may change the course of the reaction. The general picture of the concerted nature of these cycloadditions was even questioned. A controversy evolved between Huisgen and Firestone where the latter proposed a mechanistic scheme that involved biradicals as intermediates. Long arguments were exchanged in the literature provoking experimental studies and theoretical considerations, to prove or disprove the other's point of view. In retrospect it can be stated that this exchange of ideas contributed to the clarification of the mechanistic picture of these $(4+2)$ cycloadditions. Until recently (see below) all 1,3-dipolar cycloadditions could sately be assumed to take place via concerted mechanisms. There is, however, no doubt that the mechanism of the related Diels-Alder reaction is not uniform. There are cases known where biradicals seem to be the best explanation of experimental observations, and there are also examples for zwitterionic intermediates in such reactions, although the major body of experimental results is best rationalized in terms of a concerted pathway. The discussion about concerted, synchronous, or two-stage reaction seems to be outdated. Why should the formation of two new bonds between two non-symmetrically substituted or by heteroatom substitution non-symmetrical partners, as is the case for most 1,3-dipoles, require a symmetrical transition structure? Computational chemistry has made significant contributions to this aspect in recent years. What computational chemistry, so far, cannot predict in a satisfactory way, is the influence of the solvent on the mechanism. Furthermore, due to the limited size of molecules which can be handled, many real systems cannot be calculated yet on a sufficiently high level of theory. 
Rolf Huisgen has provided many important experimental results to test and develop theoretical models. A wealth of information on regiochemistry and reactivity in 1,3-dipolar cycloadditions was accumulated in the sixties. At that time the explanations of both aspects were considered the most important problems in 1,3-dipolar cycloaddition chemistry. Long discussions took place in which orientation phenomena were tentatively, but unsatisfactorily explained by a combination of steric and electronic ("polarizability") effects. Many experimental studies were initiated, an excellent account of which Huisgen himself gives in the introductory chapter of the two-volume book on "1,3-dipolar cycloaddition chemistry", edited by A. Padwa. While steric effects were less controversial, as most chemists are trained to think of molecules as space-filling objects, the nature of the electronic effect remained obscure until the early seventies.

Frontier molecular orbital (FMO) theory provided a solution to this problem. This model can ratıonalize reactivity and regioselectivity in cycloadditions without requiring the intermediacy of diradicals or zwitterions. Its drawback is that the application in this context is based on the assumption of concertedness and that it does not allow a clearcut decision whether the reaction occurs in a concerted or stepwise manner. As most 1,3-dipolar cycloadditions, for differing examples see below, are best described as concerted cycloadditions the FMO model can display its elegance and simplicity.

The relative arrangement of HOMOs and LUMOs in 1,3-dipole and dipolarophile leads to three types of reactivity patterns. The first one is governed by the interaction of $\mathrm{HOMO}_{\text {dipole - }}$

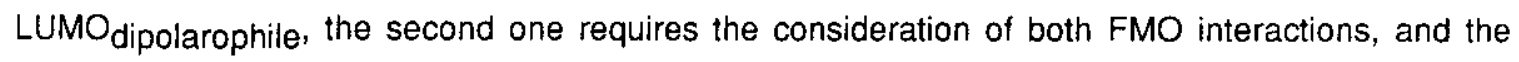
third one is rationalized in terms of a reactivity determining $L U M O_{\text {dipole }}-H O M O_{\text {dipolarophile }}$ interaction. It did not take Rolf Huisgen long to realize the potential of the model. He had already prepared the ground for it by providing systematically rate data for quite a number of 1,3-dipolar cycloadditions. In particular, the existence of U-shaped curves in the reactivity sequence, i.e. the experimental observation that some 1,3-dipoles, for instance phenyl azide, react fast with both electron-deficient and electron-rich olefins, had been established by his group and could now be explained easily in terms of the Type II reactivity pattern. Much of the experimental evidence to 
confirm the FMO model was already present at the time when it was developed, but more evidence was provided by Rolf Huisgen. In an elegant study he showed that substituents at the diazoalkane skeleton can change the reactivity behavior of this 1,3-dipolar system from Type I, over Type II to Type III of the FMO classification. A nice account of this work is given in the first chapter of the book on "1,3-dipolar cycloaddition chemistry", edited by A. Padwa.

Rolf Huisgen's capability to make use of a model which he had found suitable for his purpose is masterly displayed in the analysis of regiochemistry by FMO theory. Houk had proposed a simple explanation of regiochemistry on the basis of the magnitude of the eigenvector coefficients at the reacting atoms in the dominating HOMO - LUMO interaction. This model, based also on the assumption of a concerted cycloaddition, worked amazingly well in many cases. The attempts to put it on a quantitative basis by correlating differences in the magnitude of the eigenvector coefficients with ratios of regioisomers in 1,3-dipolar cycloadditions have not led to satisfactory results. Too sımple is the model that it might be capable of explaining such subtle differences in free energy of activation which govern the formation of one, the other, or a mixture of both regioisomers. The perserverance, one of Rolf Huisgen's special qualities, by which he persued the quantitation of the model brought important insights in the scope and limitations of such a simple theory as the FMO approach The author's present opinion on the explanation of regioselectivity, as discussed in a number of publications, is that the magnitude of the eigenvector coefficients in the FMOs may provide a reasonable explanation as long as other, in particular non-covalent interactions remain constant. Most prominent among these are non-covalent repulsions (closed-shell repulsions) and polar interactions. In principle, this is not amazing, as one or two FMO interactions are just one or two of many interactions which are present in a molecular complex.

Several 1,3-dipoles can cyclize to three-membered rings by an electrocyclic reaction or can be generated by an electrocyclic ring opening. Aziridines were among the first systems to be studied in the sixties Here, two types of pericyclic processes are combined, the electrocyclic reaction and the 1,3-dipolar cycloaddition as a means of trapping the short-lived intermediates. In classic work Rolf Huisgen not only established the stereochemical course of the thermal and photochemical ring 
opening of the cis-trans isomeric dimethyl 1-arylaziridine-2,3-dicarboxylates, but also showed that the mutual thermal interconversion of the open-chain azomethine ylides could be suppressed by 1,3dipolar cycloadditions to reactive dipolarophiles like dimethyl acetylenedicarboxylate (DMAD) (scheme 10). This study is a powerful confirmation that both reactions, the electrocyclic ring opening of the substituted aziridine and the subsequent 1,3-dipolar cycloaddition, occur acccording to the rules of conservation of orbital symmetry.

Scheme 10

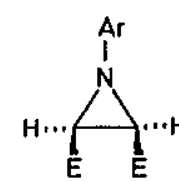

cis

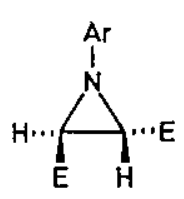

trans
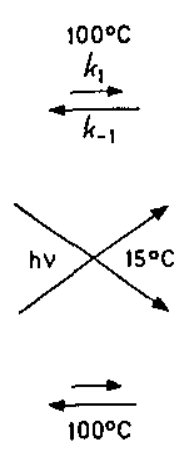

$\mathrm{Ar}=\mathrm{C}_{6} \mathrm{H}_{4} \mathrm{OCH}_{3}-(4), \quad \mathrm{E}=\mathrm{CO}_{2} \mathrm{CH}_{3}$<smiles>[CH2]/C(=C\F)[N+]([ClH+])=CF</smiles><smiles>CCO[Mg]</smiles>

trans

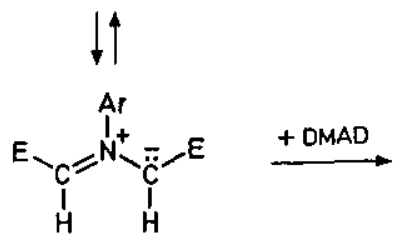

cis<smiles>[3H][C@H]1C(F)=C(F)C([CH])([CH])N1[Ga]</smiles>

trans

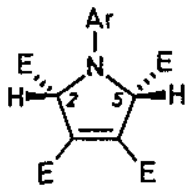

cis

Other three-membered heterocycles as precursors of substituted carbonyl ylides and thiocarbonyl ylides were oxiranes and thiiranes. The introduction of elements of the third row of the periodic table increases the number of possible 1,3-dipoles considerably. Much remains to be done in this area. Huisgen's group has studied thiocarbonyl ylides which may not only be obtained by electrocyclic ring opening of thiooxiranes but also by thermal extrusion of nitrogen from 1,3,4-thiadiazolines. These investigations are of special relevance insofar as they demonstrate for the first time that 1,3-dipolar cycloadditions may also be non-concerted under special conditions (scheme 11). The sterically very different ends of the 2,2,4,4-tetramethyl-3-thioxocyclobutanone-S-methylide are one factor favoring one-bond formation. However, an electronic contribution must also be present. Huisgen relates the latter to a small gap between $\mathrm{HOMO}_{\text {dipole }} \mathrm{LUMO}_{\text {dipolarophile }}$ and a large separation of the 
second HOMO - LUMO pair. It has to be seen whether these are general conditions which favor twostep processes via zwitterions. To my knowledge, no 1,3-dipolar cycloaddition has been reported where a biradical is detected as a transient intermediate. This may have something to do with the presence of atoms of different electronegativity in 1,3-dipoles which should favor zwitterions over biradicals as intermediates. The important result of Huisgen's study concerning the cycloaddition of 2,2,4,4-tetramethyl-3-thioxocyclobutanone-S-methylide is the unequivocal demonstration of a twostep mechanism, i.e. a mentally existing barrier that all dipolar cycloadditions take place via a uniform concerted mechanism has been crossed.

\section{Scheme 11}
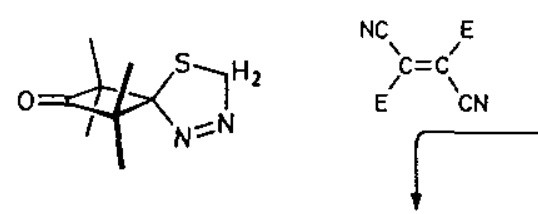<smiles>C=[Si]=C1C(C)(C)C(=O)C1(C)C</smiles>

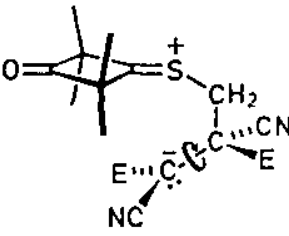

$E=\mathrm{CO}_{2} \mathrm{CH}_{3}$

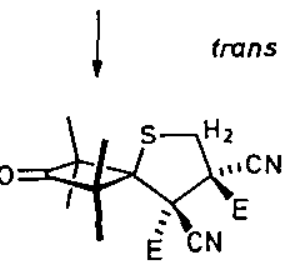

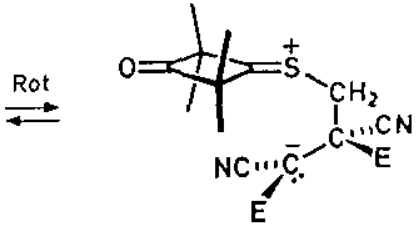

cis

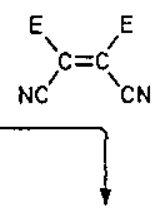<smiles>CCC</smiles>

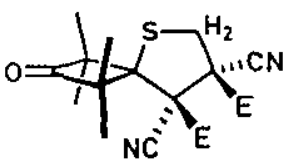

$\begin{array}{lllll}\text { Dimethyl Dicyanofumarate } & \rightarrow & 61 & : & 39 \\ \text { Dimethyl Dicyanomaleate } & \longrightarrow & 25 & : & 75\end{array}$

In an earlier study Huisgen had attempted to carry the precision of experimentally determined stereospecificities as far as possible. This is of relevance because it provides a lower limit for the difference in free energy of activation for a concerted vs, a two-step cycloaddition. The cycloaddition of diazomethane to methyl tiglate afforded quantitatively the cis-dimethyl-1-pyrazoline (scheme 12). 
A stereospecificity of $>99.997 \%$ was reported, certainly a world record in precision. It shows that the difference in free energy of activation for the two processes must be greater than $6 \mathrm{kcal} \mathrm{mol}^{-1}$.

Scheme 12<smiles></smiles>

Stereospecificity

$>99.997 \%$

Studies in recent years concern the high dipolarophilic activity of thiones. In kinetic studies on the cycloadditions of the nucleophilic 1,3-dipole diphenyldiazomethane (high lying HOMO) the CSdouble bond proved to be more reactive than tetracyanoethylene (TCNE) by a factor of 115 , although TCNE is in general the most reactive double bond for this type of 1,3-dipole. The numbers in scheme 13 represent $10^{2} \times \mathrm{k}_{2}$ obtained in DMF at $40^{\circ} \mathrm{C}$, respectively in $\mathrm{CDCl}_{3}$ when marked with an asteric. A simple and convincing explanation for these observations remains to be found (scheme 13).

Scheme 13<smiles>N#CC(C#N)=C(C#N)C#N</smiles>

$3980^{*}$<smiles>S=C1c2ccccc2-c2ccccc21</smiles>

450000<smiles>O=C1C=CC(=O)O1</smiles>

79<smiles>O=C1CC(=S)CC1=O</smiles>

$66800^{*}$<smiles>[R]C#CC(=O)O[Na]</smiles>

10200

$$
\mathrm{R}-\stackrel{\mathrm{H}}{\mathrm{C}}=\underset{\mathrm{H}}{\mathrm{C}}-\mathrm{CO}_{2} \mathrm{C}_{2} \mathrm{H}_{5}
$$

8.1

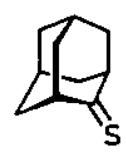

101

It seems to be an impossible task to give justice to all individual stones of the mosaique which represents Rolf Huisgen's work on 1,3-dipolar cycloadditions. In such a short survey some aspects may have been overemphasized, whereas others may have been underrated due to the author's 
predilection. Rolf Huisgen has written a number of reviews where interested readers may find resumés on individual subjects. The entire width of the field of 1,3-dipolar cycloadditions which has been proven to be so fruitful over the last three decades cannot be presented in such an account. Already ten years ago the flood of information on these reactions had become so big that only a twovolume book, the one edited by A. Padwa, was able to describe all facets of the reactions of individual 1,3-dipoles. This book also gives an excellent introduction to the field by Rolf Huisgen. The scientific success of the mix of mechanistic investigations and synthetic applications may never have been demonstrated so convincingly as in the area of 1,3-dipolar cycloadditions.

Although 1,3-dipolar cycloadditions constitute Rolf Huisgen's most important contribution to heterocyclic chemistry this short essay on his achievements in organic chemistry should not be closed before some of his other investigations have been mentioned briefely.

The area of cycloaddition chemistry in general has profitted from Huisgen enormeously. He developed not only 1,3-dipolar chemistry but made also important contributions to the field of $(2+2)$ cycloadditions. These investigations led to a profound mechanistic understanding of the reactions of donor- with acceptor olefins which, in combination with Paul Bartlett's seminal investigations on the biradical $(2+2)$ cycloadditions have led to a comprehensive picture of this type of reaction. In this context the studies on cycloadditions of ketenes should be mentioned. They provided impulses for the further development of the orbital symmetry rules for $(2+2)$ cycloadditions.

Investigations on electrocyclic reactions had started in Huisgen's group before the term was coined by Woodward and Hoffmann. Once the rules of orbital symmetry had been published some of the most elegant confirmations were to come from the Munich laboratory (see above).

At an early stage of his scientific career Rolf Huisgen was involved in studies of medium-sized ring effects. Benzyne chemistry was still another playground. Every topic picked up by Rolf Huisgen reflects his exemplary way of thinking and performing experiments. In all cases he contributed significant results such that his name will remain connected to these areas. 
The concluding remark made by Ken Houk and the author in "Rolf Huisgen and 1,3-dipolar cycloadditions" in ref.2) characterizes Rolf Huisgen and his approach to chemistry perfectly and shall be repeated here:

" Indeed, Huisgen's brillance and mark as a complete chemist is manifest in his discovery and investigations of the 1,3-dipolar cycloaddition: from an initial concept he produced farreaching experimental reality, and from mechanistic study came profound theoretical insights. Both organic theory and synthesis have been advanced immeasurably by Huisgen's science".

\section{References}

1. R. Huisgen, "Adventure Playground of Mechanisms and Novel Reactions", in the series "Profiles, Pathways, and Dreams", ed. J. I. Seeman, American Chemical Society, Washington, DC, 1994.

2. "Rolf Huisgen und sein Arbeitskreis", eine Autobiographie von Mitarbeitern, Gästen und Freunden aus Anlaß der Emeritierung, zusammengestellt von G. Boche, München 1988.

3. S. Hünig in ref. 2, p. 2.

4. P. v. R. Schleyer in ref. 2, p. 6.

5. K. N. Houk, R. Sustmann in ref. 2, p. 10. 